BULETINUL INSTITUTULUI POLITEHNIC DIN IAȘI

\section{sciendo}

DOI: 10.2478/BIPMF-2021-0007
(BULLETIN OF THE POLYTECHNIC INSTITUTE FROM IASSI)

Published by "Gheorghe Asachi" Technical University of Iasi

Volume 67(71), No. 2, 2021

Section Mathematics Theoretical Mechanics. Physics

\title{
THE ATOM OF THE PHYSICAL "REALITY" OR THE ESSENTIALITY OF THE VACUUM
}

\author{
DECEBAL VASINCU ${ }^{1}$, ALEXANDRA IULIANA SAVIUC ${ }^{2, *}$ and \\ VLAD GHIZDOVĂŢ ${ }^{3}$ \\ 1"Grigore T. Popa" University of Medicine and Pharmacy, Faculty of Dental Medicine, \\ Biophysics Department, Iaşi, Romania \\ 2“Alexandru Ioan Cuza" University, Faculty of Physics, Iaşi, Romania \\ 3"Grigore T. Popa" University of Medicine and Pharmacy, Faculty of Medicine, Biophysics and \\ Medical Physics Department, Iaşi, Romania
}

Received: April 16, 2021

Accepted for publication: May 31, 2021

Abstract. A correlation between the informational Universe and various structures of the Universe (atom, planetary systems, galaxy pairs etc.) are established. The link between these structures can be found in the concept of holography, which can be mathematically explicated through multifractality.

Keywords: informational Universe; atom; holography; multifractality.

\section{Introduction}

The ether, or the fifth element, is represented by all the "materials" that make up the universe, being, in essence, everything that exists in the space vacuum. The concept was theorized in the nineteenth century as the main medium through which light travels.

The word "ether" comes from the Greek and refers to "heaven" or "pure air". According to Greek beliefs, this is the air that the gods breathe, the equivalent of the oxygen we breathe. The ether plays an important role in the

${ }^{*}$ Corresponding author; e-mail: iuliana.saviuc@gmail.com

(C) 2021 Vasincu D. et al. This is an Open access article licensed under the Creative Commons Attribution-Non Commercial - No Dericatives 4.0 International License (CC BY-NV-ND 4.0) 
mythology of creation, according to which the beginning of the universe known to man comes from a single hatching egg, the "cosmic egg". This cosmogony is found in many civilizations of the world, with the same general concepts. For example, in the Hindu Vedas, Brahmanda is spoken of in detail - the eggshaped cosmos (brahman means cosmos and anda, egg). The egg floats in the vacuum of space and eventually breaks into two pieces, creating Dyaus Pitar, or Heaven and Prithvi, or Earth. This symbol marked not only the cosmogonies of many peoples, but also the imagination of visionary artists such as Salvador Dali.

\section{The Universal Information Matrix}

The self-structuring of matter, from the smallest scale (the subatomic one) to the largest scale (the cosmological one) represents in itself the information present everywhere (in all the structures of matter, both living and non-living). In other words, nothing that is gained through evolution is lost, but remains instead fixed in an informational structure. As any structure is part of another class of structures, up to the macrostructure called the Universe, one arrives, step by step, at the universal information matrix. Through one of the links (umbilical cords) of this chain, the biostructures are also connected to this universal information matrix. Biorhythms, the fluctuation of the functionality of all biostructures according to the periodicity of events that occur, for example, on a cosmogonic scale (in our solar system, etc.) are clarifications of such a statement (Dulcan, 2009):

i) the explosion of morbidity by strokes in years of intense solar activity;

ii) the behaviour of the Eunice worm living in the Atlantic Ocean, the fact that it detaches a part of its body, filled with gametes, exactly on the eighth night after the first full moon of early autumn, at two o'clock at night - the calendar date varies, but the phenomenon is synchronised with the moments that follow the full moon;

iii) the behavioural changes of consumers, with profound implications in economic crises (by saturating the financial markets, the commodity market, etc.), in correlation with variations in the dynamics of the terrestrial electromagnetic field, etc.

\subsection{Informational Patterns}

The concept of archetype can be translated here by the phrase general informational pattern, through which the information accumulated through the experience of each species is sedimented and from which the individual informational pattern emerges. In any biostructure whose complexity is comparable to that of the human being, with in billions of cells, each cell involving a multitude of chemical reactions per second, it would be difficult to understand, by simple guidance by the vegetative nervous system and genetic 
information, how this "universe of reactions" works, well oriented towards a biological purpose, if we do not admit the idea of informational pattern. It is very likely that the individual informational pattern will itself have the "command" in the general informational pattern. Here is "located" probably the "great attractor" of any complex structure, the hub through which they integrate into the universal information matrix, and in the particular case of the human being, the hub through which they integrate as being, species, life form, etc. (Dulcan, 2009).

\subsection{The Coherence of the "Whole" Through Individual Informational Pattern / Global Informational Pattern Connections}

As the substance in the triad that defines matter - "state", information, substance - self-structures itself on increasingly complex successive levels up to the infinite universe, so we can imagine a self-structuring on levels of succession based on informational patterns. The interdependence and integration of subsystems into systems, of systems into supersystems - and the hierarchy could continue -, is made explicit by the coherent functionality of the "whole", whether it is defined as atom, molecule, cell, organism, biocoenosis or universe.

Admitting such an approach here, we could answer many questions that do not yet have a complete answer. For example, in this manner one could understand and explain both the relative stability of species over time and the preservation of new "acquisitions" that add to their evolution, one could understand and explain the modelling of shapes, tissues according to "location" and "needs" incompletely explained by genetics, etc. From such a perspective, the universe no longer appears as an "agglomeration of disparate objects and biostructures", but as parts of a whole, between which logically determined connections are established, connections that determine its meaning, a purpose, a destiny.

From this perspective, it is easy to understand the "boom" generated by quantum mechanics in understanding and explaining microscopic phenomena on the basis of a simple wave-corpuscle duality (de Broglie's hypothesis). In such a conjecture, the "coherent" functionality of the whole, no matter what it is called, implies logically determined connections between individual informational patterns and general informational patterns, based on the mixture of states, the superposition of states, etc.

\subsection{Implicit Information, Explicit Information and their Decryption as Interaction}

The "algorithmic" functionality contained in informational patterns, either individual or general, implies the existence of implicit information at any scale. For example, if we refer to cells, biological reactions, genetic code, nervous and endocrine structures, the immune system, ion exchanges, etc., they 
all become "tools" for explaining intrinsic information. It is the information that opposes entropy.

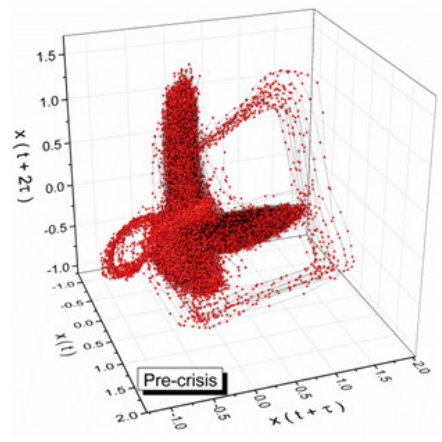

(a)

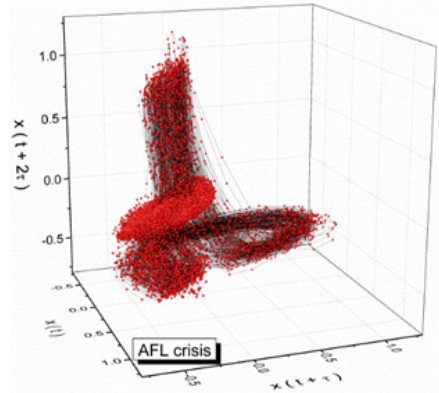

(c)

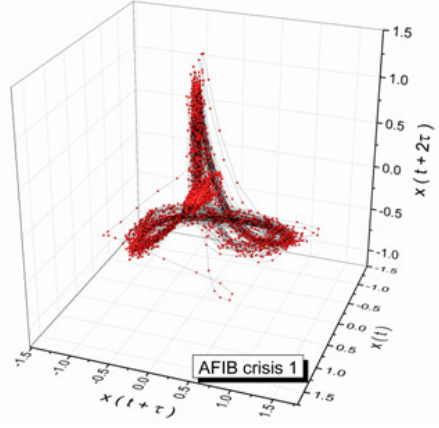

(b)

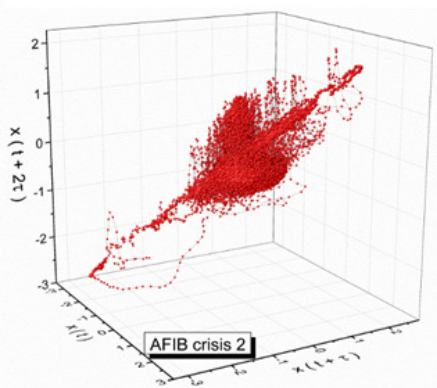

(d)

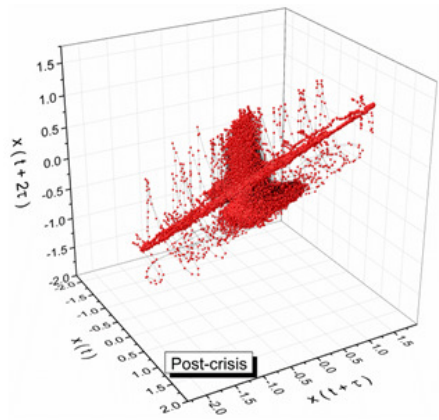

(e)

Fig. 1 - Attractors of the myocardial dynamics in the reconstructed space of states: a) pre-crisis; $b$ ) the first episode of auricular fibrillation (AFIB1); $c$ ) flutter fibrillation episode (AFL); $d$ ) the second episode of auricular fibrillation (AFIB2); $e$ ) post-crisis (according to Zală, 2019). 
At the "interface" between individual informational pattern and general informational pattern, another type of information is "manifested", namely explicit information. Thus, the limits of the functions of an individual informational pattern are exceeded and the "algorithms" contained in the default information are coordinated. Consequently, the correlation of the functions and structures of various individual informational patterns for a purpose implies, ab initio, the existence of explicit information, which transcends individual informational patterns.

In such a theoretical construction, the "interface" decrypts the structuralities and functionalities of the individual information patterns "stored" in the form of information implicit in the structuralities, and functionalities of the general information pattern stored in the form of explicit information. The result of decryption is the "interaction", usually assimilated to the concept of force, regardless of its nature, and cause of motion (Newton, 1956). We present in Fig. 1 such a decryption in the form of heart rhythm problems. The individual informational patterns are assimilated to the attractors of the myocardial dynamics in a space of states reconstructed using the lag time method, the time being determined through the method of the autocorrelation function (Cristescu, 2008; Zală, 2019).

\section{The Self-Similarity of the World. From Atoms to Planetary Nebulae Through the Holographic Universe}

Recent research and astronomical observations have shown that there exists an atomic system-stellar system analogy for the classical images of the probability density distribution of the electron in the atom (Crăciun, 2008). Moreover, it has been confirmed that Rydberg atoms (i.e., strongly energized atoms with the principal quantum number $\mathrm{n}$ of the magnitude of several hundred and therefore increasingly "classical"), currently called also planetary atoms, have a strong analogy with stellar systems.

To date, there is no theoretical gravitational model for explaining the atomobject astrophysics analogy. We can, however, offer such a model based on the fractal theory of motion (Nottale, 2011), accepting that even in the macroscopic case, probability densities do not have only the "abstract" interpretation of the location of a "punctiform" electron in one place or another in space. For example, when electrons become bound (to the atom), their mass (and charge) is "scattered" (diffused) along a structure that is isomorphic with the probability density distribution that depends on the energy state of the atom. Thus, abstract probability densities become proportional to actual mass distribution densities.

Since atom - star system analogies manifest themselves on very different scales, we can consider that the Universe has a multifractal (holographic) structure also called a self-similar cosmological paradigm. Direct observational verification of this hypothesis is difficult because only the shape of one star (the Sun) can be observed from Earth. Although the shape of the sun 
is consistent with the atom-star similarity, data on several stellar systems in the Universe are needed in order to generalize this analogy.

The difficulty of observation derives from the fact that near the stars there are "punctiform" sources that prevent the accuracy of the observations with existing telescopes. Thus, their detailed form remains unknown for the time being. However, there are solar systems (for example, so-called "planetary nebulae") that expel ("eject") their outer layers in a distribution that could be related to the initial shape (state) of the star. Obviously, in the process of expulsion some diminutions, some losses of pre-symmetries can occur, for these expanding astrophysical objects.

In support of this hypothesis come also the results obtained with the Hubble telescope, which have shown that very young planetary nebulae have structural characteristics similar to those of mature planetary nebulae. The large number of similar atomic-star systems should exclude a major influence of subjective or random factors. Moreover, considering the equations (Crăciun, 2008):

$$
E_{n}=-\frac{\mu}{2}\left(\frac{G M}{2 \sigma n}\right)^{2}=-\frac{\mu}{2}\left(\frac{v_{0}}{n}\right)^{2}
$$

where

$$
\mu=\frac{m_{1} m_{2}}{m_{1}+m_{2}}, \quad M=m_{1}+m_{2}
$$

It is possible that the relative recession velocities, $v_{n}$, in binary galaxies to be "quantified" according to the equations:

$$
v_{n}=\frac{v_{0}}{n}, v_{0}=\frac{G M}{2 \sigma}
$$

The meanings of the magnitudes involved in Eqs. (1)-(3) are given in (Crăciun, 2008). For certain binary galaxies we present as a comparison in Table 1 the observed and the "quantified" values of recession velocities.

\begin{tabular}{|c|c|c|c|c|c|}
\hline $\begin{array}{l}\text { Double } \\
\text { galaxy }\end{array}$ & $\bar{n}$ & $\begin{array}{l}\mathrm{m}_{1} \text { and } \mathrm{m}_{2} \\
\times 10^{10} M \leftarrow\end{array}$ & $\mathrm{V}_{\mathrm{obs}}\left(\mathrm{kms}^{-1}\right)$ & $\mathrm{V}_{\text {pred }}\left(\mathrm{kms}^{-1}\right)$ & $n D M \cdot 10^{67} \mathrm{Js}$ \\
\hline \multirow{2}{*}{$\begin{array}{l}\text { NGC-3958 } \\
\text { NGC-3963 }\end{array}$} & \multirow{2}{*}{1} & 12.0 & \multirow{2}{*}{72.03} & \multirow{2}{*}{72} & \multirow{2}{*}{8.0} \\
\hline & & 9.0 & & & \\
\hline \multirow{2}{*}{$\begin{array}{l}\text { NGC-4294 } \\
\text { NGC-4299 }\end{array}$} & \multirow{2}{*}{2} & 1.4 & \multirow{2}{*}{34.98} & \multirow{2}{*}{36} & \multirow{2}{*}{7.77} \\
\hline & & 8.8 & & & \\
\hline \multirow{2}{*}{$\begin{array}{l}\text { NGC-4085 } \\
\text { NGC-4088 }\end{array}$} & \multirow{2}{*}{3} & 1.3 & \multirow{2}{*}{25.04} & \multirow{2}{*}{24} & \multirow{2}{*}{8.34} \\
\hline & & 6.0 & & & \\
\hline \multirow{2}{*}{$\begin{array}{l}\text { NGC-3504 } \\
\text { NGC-3512 }\end{array}$} & \multirow{2}{*}{4} & 1.0 & \multirow{2}{*}{20.58} & \multirow{2}{*}{18} & \multirow{2}{*}{9.10} \\
\hline & & 5.0 & & & \\
\hline \multirow{2}{*}{$\begin{array}{l}\text { NGC-6542 } \\
\text { NGC-6528 }\end{array}$} & \multirow{2}{*}{5} & 2.7 & \multirow{2}{*}{15.09} & \multirow{2}{*}{14.5} & \multirow{2}{*}{8.38} \\
\hline & & 1.7 & & & \\
\hline
\end{tabular}

Table 1

Observed and Theoretical Values of Recession Velocities in Binary Galaxies 
It follows that: i) for binary galaxies there exists a maximum recession velocity of a value $v_{0}=\langle w\rangle / 2=144 \mathrm{kms}^{-1} / 2=72 \mathrm{kms}^{-1}$ (for further details, please see (Tifft, 1997); ii) there exists a gravitational type analogue of Planck's constant. It has the value $h_{g} \approx(8.3 \pm 0.2) \times 10^{67} \mathrm{Js}$ (Crăciun, 2008).

Next, we present images (Fig. 2) that support the stellar atom-system (planetary nebula) analogy. The figures on the left represent a state of energization of the atom, characterized by the quantum numbers $n, l, m$, while the photos on the right are of similar planetary nebulae. (http://www.amherst.edu/rlolders/MENU.HTM).
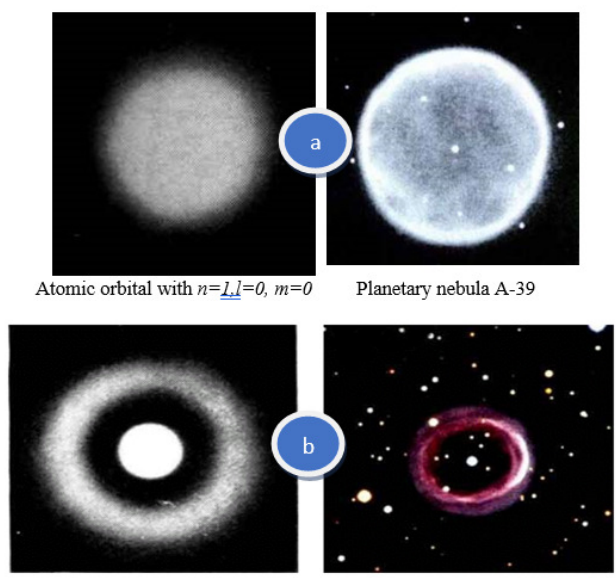

Atomic orbital with $n=2, l=0, m=0$

Planetary nebula SHARP-1
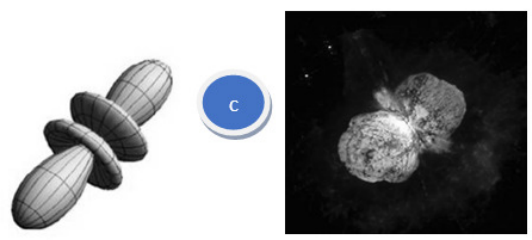

Atomic orbital with $n=4, l=4, m=0$

Planetary nebula Eta Carinae
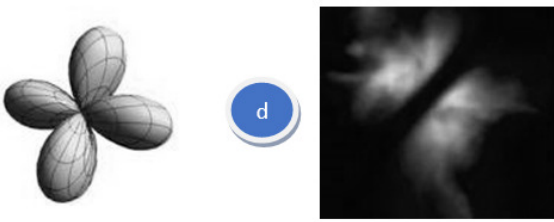

Atomic orbital with $n=3, l=3, m=1$

Planetary nebula IRAS $04302+2247$

Fig. 2 - The atom - planetary nebula analogy. 
Therefore, the process of discovering the Whole (be it the observable universe) in Nothingness (be it the common grain of sand) represents both structural and functional sequences of the hologram of this world.

Nothingness has now become the centrepiece of modern physics; it is the starting point of our universe and it also conditions its future. Nothingness, or vacuum, possesses its own energy and is by no means a nonexistence, but rather a state of hibernation of matter. Pure nothingness, as we conceive it, cannot exist, on the contrary, it is extremely complex and contains mysteries that we must decipher if we are to understand the whole universe.

\section{REFERENCES}

Crăciun P., Contributions to the Study of Gravitational Fields in the Linear Approximation, PhD Thesis: (in Romanian), Iaşi (2008).

Cristescu C.P., Nonlinear Dynamics and Chaos. Theoretical Fundaments and Applications (in Romanian), Romanian Academy Publishing House (2008).

Dulcan D.C., The Intelligence of Matter, $3^{\text {rd }}$ Ed. (in Romanian), Cluj-Napoca, Eikon (2009).

Newton I., The Mathematical Principles of Natural Philosophy (translated in Romanian by Victor Marian), Bucharest, Romanian Academy Publishing House (1956).

Nottale L., Scale Relativity and Fractal Space. A New Approach to Unifying Relativity and Quantum Mechanics, London, Imperial College Press (2011).

Tifft W.G., Redshift Quantization in the Cosmic Background Radiation Rest Frame, Journal of Astrophysics and Astronomy 18, 415-433 (1997).

Zală A., Research Regarding Interactions Between Biological Structures and Electromagnetic Fields with Applications in Balneo and Physiotherapy, $\mathrm{PhD}$ Thesis: (in Romanian), Iasi (2019).

http://www.amherst.edu/rlolders/MENU.HTM.

\section{ATOMUL „REALITĂȚII” FIZICE SAU ESENȚIALITATEA VIDULUI}

(Rezumat)

În această lucrare sunt prezentate corelații între Universul informațional și diferitele structuri ale Universului (atom, sisteme planetare, perechi de galaxii etc.). Relația dintre aceste structuri poate fi găsită în conceptul de holografie, ce poate fi explicitat matematic prin multifractalitate. 